\title{
PRESENTACIÓN DE LA INFORMACIÓN EN ENTREVISTAS DE LA RADIO PÚBLICA ESPAÑOLA ${ }^{1}$
}

\author{
Esperanza Morales López \\ Universidad de La Coruña
}

\begin{abstract}
«[...] No quedan en el lenguaje palabras y formas neutrales, de 'nadie': el lenguaje se ve totalmente malversado, recorrido por intenciones, acentuado. Para la conciencia que vive en él, el lenguaje no es un sistema abstracto de formas normativas, sino una opinión plurilingüe concreta acerca del mundo. Todas las palabras tienen el aroma de una profesión, de un género, de una corriente, de un partido, de una cierta obra, de una cierta persona, de una generación, de una edad, de un día, de una hora. Cada palabra tiene el aroma del contexto y de los contextos en que ha vivido intensamente su vida desde el punto de vista social; todas las palabras y las formas están pobladas de intenciones» (Bakhtin, 1981: 110).
\end{abstract}

1 Este trabajo se enmarca dentro del Proyecto ADPA (Análisis del discurso público actual), que se está desarrollando en el Área de Linguiística General de la Universidad de La Coruña, financiado por la Xunta de Galicia desde 1995 (última referencia XUGA 10402B98). 


\section{INTRODUCCIÓN}

La entrevista es un tipo de discurso público o institucional ${ }^{2}$, ampliamente extendido tanto en el periodismo profesional como en la televisión y la radio, cuya principal función comunicativa es la transmisión de información u opinión de ciertas figuras públicas o expertos en una determinada materia ante un tipo de audiencia (Heritage y Greatbatch, 1991: 106).

Desde el punto de vista funcional, la entrevista puede explicarse a partir de la noción de acto de habla como una actividad que cumple diversas funciones sociales (Heritage y Roth, 1995: 2; Levinson, 1983: 275, y 1992). Los dos roles sociales que intervienen en la misma, entrevistador ( $E R$, a partir de ahora) y entrevistado $(E O)$, contribuyen al desarrollo del tópico iniciado a través de un intercambio de actos de habla de pregunta-respuesta; de esta manera se produce una negociación a lo largo del proceso interactivo. En este artículo me voy a referir exclusivamente a la entrevista bipartita entre un único entrevistador y un único entrevistado ${ }^{3}$.

La entrevista, como cualquier otro tipo de género o subgénero presente en los medios de comunicación social actuales, necesita cumplir ciertas funciones generales que son comunes también a otros géneros periodísticos: suscitar el interés de la audiencia ante el tema en cuestión, ofrecer una actitud de neutralidad y veracidad ante los acontecimientos que se citan, entre otros. Sin embargo, la ampliación del contexto de los medios de comunicación en la actualidad y la diversificación de sus posibilidades han provocado la aparición de nuevos subgéneros dentro de la entrevista misma: entrevista informativa, interpretativa, de carácter, etc. (Balsebre y otros, 1998; Cantavella, 1996). La especificidad de tales subgéneros vendrá determinada por la finalidad concreta que el entrevistador (o el medio de comunicación para el que trabaja) persiga con tal entrevista: impacto de actualidad, confirmación de declaraciones previas, petición de la opinión del entrevistado, mayor conocimiento del personaje entrevistado, etc.

2 Existen definiciones más modernas de lo que es un discurso público (véase, por ejemplo, Agar, 1985: 147); sin embargo, la ofrecida por la antigua retórica aún es, en un sentido amplio, relevante: el discurso que tiene lugar en el forum, el lugar donde se discuten y debaten los asuntos públicos. Lo que sucede es que el concepto de forum necesita ser ampliado para incluir nuevas formas, canales y lugares de comunicación.

${ }^{3}$ En un trabajo anterior (Morales López, 1995) ya presentábamos, aunque de manera bastante incipiente, algunas de estas ideas. 
En este trabajo, me voy a referir a un tipo de entrevista corta que se desarrolla en el interior de un informativo diario de la radio pública española; la franja horaria es una de las de mayor audiencia matinales. Por tanto, es importante destacar dos características en este tipo de entrevista, como exponemos a continuación.

Por un lado, se trata de un tipo de interacción que se inserta en otro género más amplio, el de un informativo diario. Este hecho determina que el tema de la entrevista se refiera normalmente a una de las noticias más importantes de ese momento. La función comunicativa de esta entrevista se reduce principalmente a la confirmación de la veracidad de tal noticia, de su importancia o actualidad (debido a que la información que se aborda en tal entrevista ya ha sido presentada con anterioridad en forma de titulares más o menos desarrollados); o bien a conseguir la opinión del entrevistado acerca de la noticia en cuestión.

Por otro lado, se realiza en un medio de comunicación de una entidad que es financiada por el Gobierno y, por tanto, de carácter distinto a otro tipo de medios de comunicación privados. Si repasamos la historia de la radio y la televisión públicas en España, en el periodo de transición de la dictadura a la democracia, el sistema de ente público que se adoptó fue similar al modelo francés. Así, el Director de Radiotelevisión Española es elegido por el partido que gobierna en un determinado momento, y los miembros de su Consejo de administración lo son por el Parlamento, en proporción a la representación política que en esa legislatura ostentan los diferentes partidos. En consecuencia, este medio de comunicación depende considerablemente del partido gobernante de turno, ya que refleja siempre la distribución de fuerzas políticas elegidas democráticamente. Esto explica también las continuas críticas de manipulación que ha recibido este ente público desde su creación (Bustamante, 1989: 80 y 85; para una comparación del modelo francés de radiotelevisión pública con el británico, véase Kumar, 1975).

En un contexto legislativo como el expuesto, es legítimo plantearse el tema de cómo se ejerce el poder en un momento dado a través de las distintas prácticas simbólicas que se generan desde este medio; asimismo nos podemos preguntar también en qué medida son indicativas de la perspectiva de quien ostenta tal poder.

Sin embargo, un medio de titularidad estatal necesita también ejercer el equilibrio necesario para armonizar los diversos intereses entre unos grupos y otros, defendiendo al mismo tiempo la estabilidad 
democrática. En esta dirección, Hall (1973: 87-88) señala que otra de las funciones del trabajo periodístico (o de los medios de comunicación en general) es la de actuar como mediación entre lo público y lo privado, entre lo nuevo y lo tradicional; entonces, en este caso, ya no nos encontramos con la inclinación de los editores de noticias hacia una posición política determinada, sino con un sesgo informativo que tiende a preservar los valores que representan el consenso entre los diferentes grupos o instituciones. Tomando en consideración esta perspectiva de Hall, otro aspecto que será objeto de nuestro análisis es la consideración de en qué medida las diferentes construcciones discursivas contribuyen a conseguir este equilibrio de fuerzas en la defensa del statu quo.

Desde el punto de vista metodológico, en este trabajo parto del presupuesto de que el análisis de las diferentes prácticas discursivas no sólo es un medio de observación de los condicionamientos sociales de quienes los han generado, sino sobre todo un medio privilegiado de creación de tales condicionamientos. Es decir, los hablantes (debido a los roles sociales que representan en cada interacción discursiva) están constreñidos por ciertas dependencias sociales, culturales o políticas que les vienen dadas por razón de su papel social. Sin embargo, también es cierto que es en el proceso interactivo que se desarrolla entre los interlocutores en el proceso discursivo donde se construyen de hecho tales relaciones y se pueden romper los moldes impuestos con anterioridad. De esta manera, tal como ha sido defendido en la tradición de la Sociolinguística interaccional (Gumperz, 1982) y la Etnografía de la comunicación (Duranti, 1994: 138-139, entre otros), las prácticas lingüísticas no sólo reflejan relaciones de poder, sino que ellas mismas las constituyen en el proceso interactivo.

\section{MODOS DE PRESENTARSE LA INFORMACIÓN}

En este trabajo, mi objetivo es el estudio de la construcción de la noticia en el desarrollo de la entrevista a partir de las diferentes prácticas simbólicas que he denominado de indirección. Es a través de tales prácticas como el ER intenta conseguir, por un lado, un equilibrio entre la constricción que el subgénero mismo le impone y, por otro, la salvaguarda de los intereses del autor de este medio público. 
Más concretamente, debido a que se trata de una entrevista incluida en un informativo diario, ésta queda limitada por la obligatoriedad de aludir en ella a una de las noticias de actualidad (normalmente una de las más sobresalientes del día). Sin embargo, este hecho puede entrar en colisión con las intenciones concretas o la posición ideológica de este medio como entidad pública (tal como se concreta en el contexto español que ya hemos explicado). Por este motivo, el ER recurre a la construcción de diferentes estrategias a través de las cuales intenta conseguir un equilibrio entre ambos aspectos.

A estas prácticas o estrategias es a lo que he denominado procesos indirectos en la presentación de la información; son construcciones lingüísticas y discursivas cuya intención comunicativa no se corresponde con su significado denotativo o explícito. Constituirían el sesgo intencional consciente o inconsciente que el ER construye durante el proceso de negociación del tópico de la entrevista.

Tales prácticas simbólicas pueden ser enormemente variadas, constituyéndose a partir de las distintas estructuras lingüísticas, discursivas, retóricas o argumentativas (Thompson, 1990; Van Dijk, 1995 y 1998 , entre otros). Las funciones que esta diversidad de prácticas cumple en los distintos ejemplos de entrevistas que analizaremos las hemos agrupado en dos: por un lado, defensa de la imagen del ER en la presentación de la noticia, ante una posible amenaza que pudiera ocurrir; por otro lado, deslegitimización de personas o instituciones con las que no se está de acuerdo o no se comparten los mismos puntos de vista. Veamos estos aspectos más detenidamente.

\subsection{Defensa de la imagen}

La noción de imagen, definida por Brown y Levinson (1987: 1, 13), a partir de los trabajos de Goffman (1971, entre otros), se presenta como un rasgo universal de todas las relaciones humanas; consiste o bien en el deseo de toda persona de no verse impedida de hacer algo (lo que constituiría la imagen negativa), o bien en el deseo de ser reconocida por sus interlocutores (en este caso, nos referimos a la imagen positiva). En relación con la defensa de la imagen, Brown y Levinson (1987) señalan cómo los aspectos de cortesía, deferencia y tacto adquieren una relevancia especial en aquellos momentos que, social- 
mente hablando, pueden significar una amenaza para la imagen del individuo, considerado tanto una persona que a título privado realiza una acción determinada como un actor social que representa a una institución concreta y cuyos intereses tiene que velar y salvaguardar.

En el discurso que nos ocupa en este trabajo, cuando el equilibrio entre la fidelidad a una narración objetiva de los hechos y la defensa de los intereses propios es una tarea difícil, el entrevistador tiende a construir diversas estrategias que contrarresten la amenaza potencial que pudiera sobrevenir: amenaza por la alusión a noticias políticamente conflictivas para la institución de la que depende ese medio público (tal como mostrará el ejemplo 1) o bien para otras instituciones democráticas (ejemplo 3); o amenaza por la crítica de incumplir el imperativo democrático de igualdad en el tratamiento mediático de los diferentes grupos políticos (ejemplo 2); entre otras posibilidades. Estos ejemplos, encabezados con una explicación de las prácticas discursivas que el ER construye en el proceso interactivo, constituyen el tema de los siguientes subapartados.

\subsubsection{Ausencia de contexto y universalización}

Hall (1973: 91-3) señala que una tendencia general de la prensa diaria y una práctica que se está extendiendo a la radio es la presentación de la «actualidad sin contexto». Este hecho tiene una importante repercusión en la radio, en donde la audiencia es socialmente más diversa $\mathrm{y}$, por tanto, puede incluir un amplio grupo de población con niveles de alfabetización más bajos (aspecto que sucede mucho menos en la prensa escrita). De esta manera, a unos oyentes con menos instrucción en general se les ofrecen una serie de noticias sin información contextual que las enmarque, con las consiguientes repercusiones para la adecuada comprensión de los hechos que se relatan.

Esta tendencia puede ser explotada también en un determinado momento desde el punto de vista ideológico con el fin de contribuir a la presentación intencionada de una noticia; esto es lo que parece sugerir el ejemplo 1.

En el desarrollo discursivo de la entrevista de este ejemplo, el ER acude también al recurso de la universalización. Esta estrategia supone un cambio de marco en el desarrollo del tópico, de tal modo que la 
narración de un determinado suceso se traslada desde la presentación particular de los hechos a una presentación más general con una intención determinada (Thompson, 1990: 61). Esta intención suele ser comunicativamente diversa, pero, desde el punto de vista ideológico, puede relacionarse —entre otras muchas posibilidades - con los deseos del hablante de pretender justificar determinadas actuaciones o, como el caso que nos ocupa, una manera de aludir a una información que resulta conflictiva políticamente.

\section{EJEMPLO 1}

Entrevista a Pascual Sala, Presidente del Consejo General del Poder Judicial, a raíz de la noticia de la designación del nuevo Fiscal General del Estado. El Fiscal anterior había tenido que dimitir debido a ciertas irregularidades cometidas en su nombramiento por el Gobierno Socialista entonces en el poder (2-6-1994).

Titular: «El Pleno del Consejo General del Poder Judicial apoya por unanimidad la designación de Carlos Granados como Fiscal General del Estado».

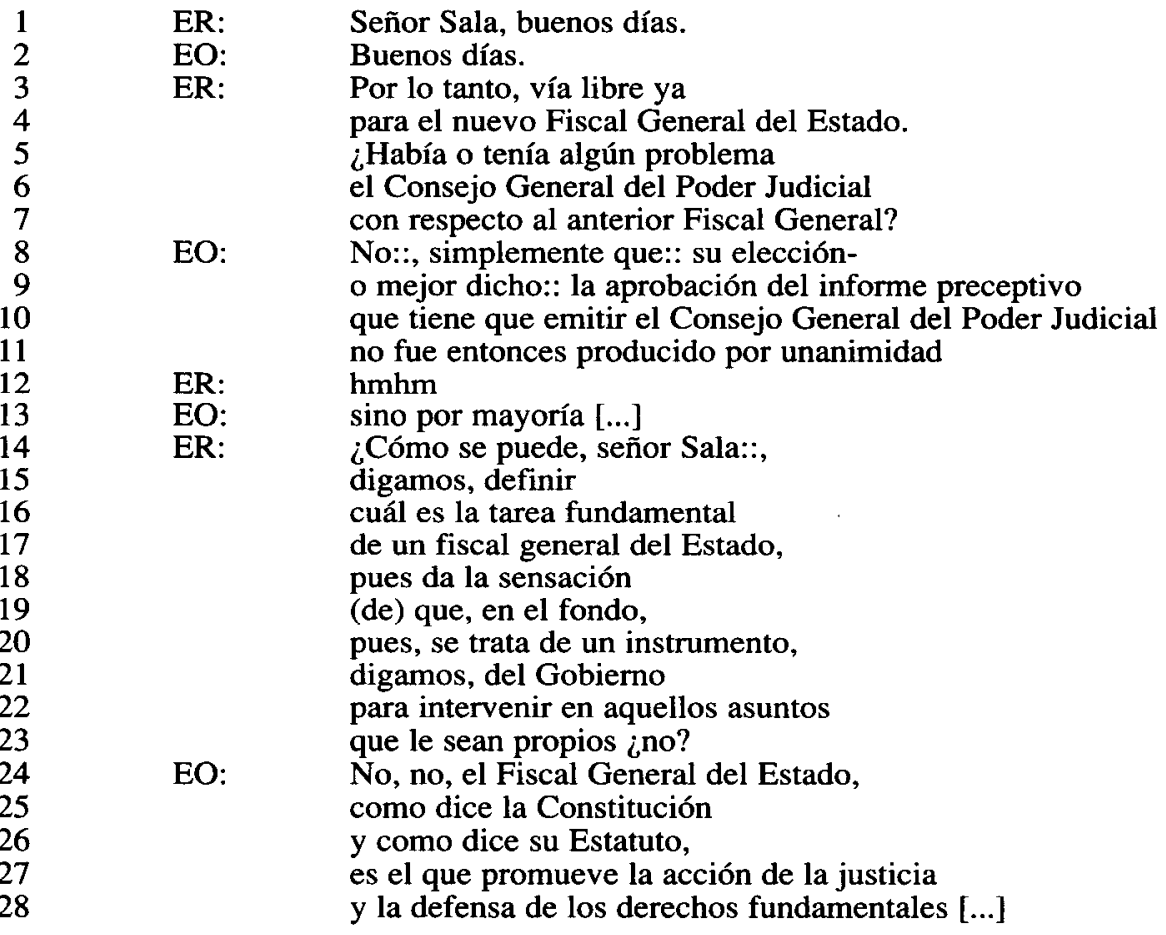

En el titular así como en la paráfrasis del mismo que se realiza en las líneas (3)-(4), la noticia se presenta como un nombramiento ruti- 
nario, sin aportar otra información adicional sobre el hecho. Sin embargo, el nombramiento del Fiscal General en cuestión fue un tema muy polémico en aquel momento, además de complejo jurídicamente; la historia se remonta a dos años antes de la publicación de la noticia, cuando se produjeron ciertas irregularidades en el nombramiento del anterior Fiscal General por parte del Gobierno. Después de un proceso judicial para determinar la legalidad del hecho, el Tribunal Constitucional anuló el nombramiento del anterior Fiscal y hubo que proceder dos años más tarde a la propuesta de otro (el señor Granados, que es a quien se refiere la noticia del día). Esta complejidad técnica, junto a la lejanía del hecho, hace prever que al público en general le resultará difícil recordar el suceso con todos sus detalles.

Un aspecto que, en mi opinión, contribuye a determinar que no se trata únicamente de un ejemplo más de la tendencia general de la prensa y la radio actuales a obviar el contexto de una noticia, sino de una construcción discursiva intencionada, es la ambigüedad con la que el ER construye su pregunta siguiente. Con el adjetivo indefinido algún en la línea (5), el ER presenta el problema como desconocido para él mismo, consiguiendo así distanciarse de los hechos. La inverosimilitud de este desconocimiento de los hechos reafirma la hipótesis de que este recurso, junto con la falta de alusión a los hechos contextuales, puede considerarse en su conjunto un constructo indirecto intencional por parte del ER en la presentación de una noticia.

En la segunda pregunta, que comienza en la línea (14), el ER realiza un cambio de alianzas hacia posiciones más generales en la referencia a esta noticia. Esta estrategia se incluye en la primera de las dos subunidades de que se compone el turno del ER, líneas (14)-(17), y que consiste en la pregunta sobre la función general del Fiscal General. En la segunda parte del turno del ER, líneas (18)-(23), se inserta un comentario personal sobre este hecho.

Este comentario personal se refiere a la controversia que rodeó la designación del anterior Fiscal General, hecho que se presenta discursivamente como información secundaria; contribuye a esto último la construcción sintáctica subordinada en las líneas (18)-(23), que resulta, además, semánticamente ambigua: «... para intervenir en aquellos asuntos que le sean propios» en las líneas (20)-(23). Lo que el término «propios» significa no es nada claro.

El comentario incluye, además, cuatro expresiones o partículas que funcionan como atenuadores (hedges) cuya función es minimizar su fuer- 
za ilocutiva: da la sensación, en el fondo, pues y digamos. Finalmente, el uso de dos construcciones impersonales con se, en las líneas (14) y (20), refuerza aún más la fuerza ilocutiva de este acto de habla.

Este ejemplo, pues, nos muestra que el uso de las dos estrategias mencionadas resultan ser sendas construcciones discursivas ligadas a una intencionalidad ideológica determinada; el análisis realizado apunta a dos estrategias indirectas creadas por el ER para referirse a unos hechos que ineludiblemente suponen cierto coste político, ya que estos hechos implican una actuación ilegal del Gobierno. Con ello, el ER consigue tanto minimizar la imagen negativa de la institución que representa como minimizar las críticas que pudiera recibir él mismo $\longrightarrow$ el equipo de redacción-, por parte de los grupos de la oposición política, por el hecho de no haber dado suficiente cobertura informativa a estos hechos.

\subsubsection{Heteroglosia}

Este término proviene de las discusiones de Bakhtin (1981: 93ss.) sobre el discurso novelístico. Específicamente, heteroglosia se refiere al fenómeno de la combinación de múltiples voces, discursos o lenguajes en el género novelesco, a través de los cuales se comunica gran parte de la información que el autor quiere transmitir. Este fenómeno también está presente en la interacción cotidiana para transmitir cualquier tipo de información; sin embargo, los recursos linguíísticos e interpretativos presentes en la conversación espontánea son mucho menos complejos. En el caso de discursos con implicaciones ideológicas, la utilización de este procedimiento adquiere mayor complejidad semántica e interpretativa.

En las entrevistas analizadas, este recurso discursivo sirve también al ER para avanzar en la negociación del significado. Con él consigue construir su propio papel de participante neutral y logra ponerse a salvo ante las críticas que recibirá de la oposición de parcialidad en el tratamiento de la noticia en cuestión (y que aparecen explícitas en la entrevista misma). Veamos el siguiente fragmento:

\section{EJEMPLO 2}

Entrevista a José Bono, miembro del Partido Socialista en el poder. El tema de la entrevista es la aparición de información relativa al caso GAL (un 
asunto de terrorismo de estado investigado por la justicia de ese momento), a raíz de nuevas revelaciones de ciertos personajes públicos, como el General Sáenz de Santamaría (26-1-1996).

ER: $\quad[\ldots]$ En cualquier caso, señor Bono, por seguir un poco con todo este asunto del GAL, ¿qué es lo que está pasando exactamente con todo eso, con estas nuevas revelaciones, vamos a ponerlo así, en relación con este hecho, tantos años después,

el General hablaba de los años ochenta, etcétera? ¿qué está pasando?

EO: Lo que ocurre probablemente es que el Partido Popular, o algunos de sus dirigentes, después de su congreso,

han creído que con el tema del terrorismo podrían ganar algunos votos.

Ese es mi criterio.

Y por eso Aznar anuncia aquello de que si gana las elecciones, - si pierde no-. si gana dice que va a «pasar página» en el tema del GAL.

Con lo cual, lo primero que hay que preguntarse es ¿y si pierden? ¿entonces no van a pasar página? ¿van a seguir en la página que están ahora, incidiendo? [...] Hay gentes dispuestas a ver en- en el campo del adversario muchos defectos,

y a disculparlos en el propio. Señor Bono, ¿cree usted que habría que pasar página también en este caso GAL o que::

hay que seguir investigando?

EO: $\quad$ Mire [...] la que hay que pasar [es] la página de ETA [...] [El ER despide al señor Bono y, después de una serie de referencias a diferentes políticos implicados en el caso GAL, inicia una nueva entrevista en relación con este mismo tema con Álvarez Cascos, un miembro destacado del Partido Popular].

ER: $\quad[\ldots]$ Hablemos de esta polémica, que usted conoce perfectamente. Bueno, ¿qué pasó, por fín, en relación con la desaparición de la «Comisión GAL»? ¿Tenían, como dicen y como les acusan desde el Partido Nacionalista Vasco y desde el Partido Socialista, tenían ustedes miedo

\section{EO: $\quad$ a mí lo primero que me preocupa} y escuchando el programa esta mañana me ratifico, es que en estos momentos se esté organizando una especie de operación de apología del GAL [...] [...] 


$\begin{array}{lll}51 & \text { ER: } & \text { Por último, señor Álvarez Cascos, } \\ 52 & & \text { el controvertido asunto de pasar página, } \\ 53 & & \text { a lo mejor no se ha entendido bien } \\ 54 & & \text { lo que ha querido decir José María Aznar. } \\ 55 & & \text { iEstarían ustedes dispuestos a- } \\ 56 & & \text { a ese paso de página en caso de ganar las elecciones? } \\ 57 & \text { EO: } & \text { Pero yo creo que lo que ha dicho José María Aznar [...] }\end{array}$

Este diálogo nos muestra un ejemplo de heteroglosia entre discursos producidos en diferentes ocasiones. Una expresión pronunciada por Aznar en un determinado espacio político (la de «pasar página») sirve a su vez a un miembro de la oposición, José Bono, para construir su crítica a la actuación del partido de la oposición en el caso GAL -líneas 22-27- La expresión en cuestión formaba parte de la precampaña política del líder del Partido Popular ante las elecciones generales ya próximas (que de hecho se celebraron dos meses más tarde, en marzo de 1996) y algunos la entendían como una estrategia electoralista que prometía, en el caso de ganar este partido las elecciones, promulgar una cierta amnistía para los inculpados en el caso GAL.

Con el fin de seguir avanzando en el tratamiento de este tema, el ER retoma también esta misma expresión tanto en su pregunta al representante del Partido Socialista, en las líneas 31-35, como al representante del Partido Popular, en las líneas 51-54. Con este procedimiento, el ER consigue aludir a un tema controvertido y que está sirviendo de arma política entre los diferentes partidos en la precampaña electoral, pero sin que apenas represente amenaza para su imagen. Gracias a este recurso construye su rol de participante neutral al no podérsele atribuir la autoría de tales afirmaciones.

En la primera de las preguntas, la que dirige al señor Bono, en las líneas 31-35, es donde primeramente retoma la expresión de «pasar página» de Aznar, en torno a la cual el EO había construido toda su respuesta, en las líneas 11-30. Posteriormente, realiza una pregunta relativamente directa al señor Álvarez Cascos sobre esta polémica entre los partidos. A esta pregunta, el Sr. Cascos responde, en las líneas 46-50, de una manera que resulta bastante amenazadora para la imagen del ER: este político considera que la radio pública se está sumando a lo que él denomina «apología del caso GAL».

En consecuencia, en la siguiente y última pregunta, el ER vuelve a retomar el procedimiento de la heteroglosia para poder seguir avanzando en el desarrollo de la entrevista (líneas 51-54), pero guardando siempre su neutralidad; así, nuevamente, cita la expresión «pasar 
página» para preguntarle a su interlocutor por este hecho; utiliza incluso una expresión que funciona como atenuador con el fin de reducir la fuerza ilocutiva de su pregunta: «a lo mejor no se ha entendido bien...», en la línea 53. Así pues, con el reiterado uso del procedimiento de la heteroglosia a lo largo de la entrevista, el ER consigue dar cobertura a unos hechos, que están sirviendo a los diferentes partidos para avivar el clima preelectoral que se respira ya, pero desde una posición de neutralidad construida; por este motivo, logra que, discursivamente, quede a salvo su imagen y la del ente público que representa.

\subsubsection{Figuras retóricas}

Una de las figuras retóricas que más están siendo estudiadas en los últimos años, tanto desde la perspectiva semántica como pragmática, es la metáfora. A partir de los estudios cognitivistas que explican este recurso retórico como uno de los más importantes utilizados por la mente humana como acceso a la organización del conocimiento (Lakoff y Johnson, 1980; Lakoff, 1986), varios investigadores se han adentrado también en el análisis de la utilización de este recurso desde el punto de vista de sus funciones discursivas (Chilton e Ilyin, 1993, entre otros). El papel de la metáfora en el discurso público es múltiple: finalidad persuasiva, mantenimiento de la imagen, establecimiento de alianzas entre los participantes, deslegitimación de alguien o algo, etc.

Estas mismas funciones pueden extenderse también a otra de las figuras retóricas más similares a la metáfora, la comparación; ésta se caracteriza por la presencia en el discurso de los dos dominios implicados, el real y el figurado. El siguiente fragmento muestra un ejemplo de utilización de esta figura retórica con un gran valor creativo, cuya finalidad es también reducir la posible amenaza que pudiera provenir al aludir a una noticia que pone en tela de juicio determinadas actuaciones de una importante institución democrática. Veamos este nuevo caso:

\section{EJEMPLO 3}

Entrevista al abogado de Nicolás Redondo (un máximo dirigente sindicalista) en el «Caso de la PSV» (presunto fraude en la construcción de viviendas sociales por una cooperativa promovida por el sindicato que lidera Nicolás Redondo) (14-7-94). 


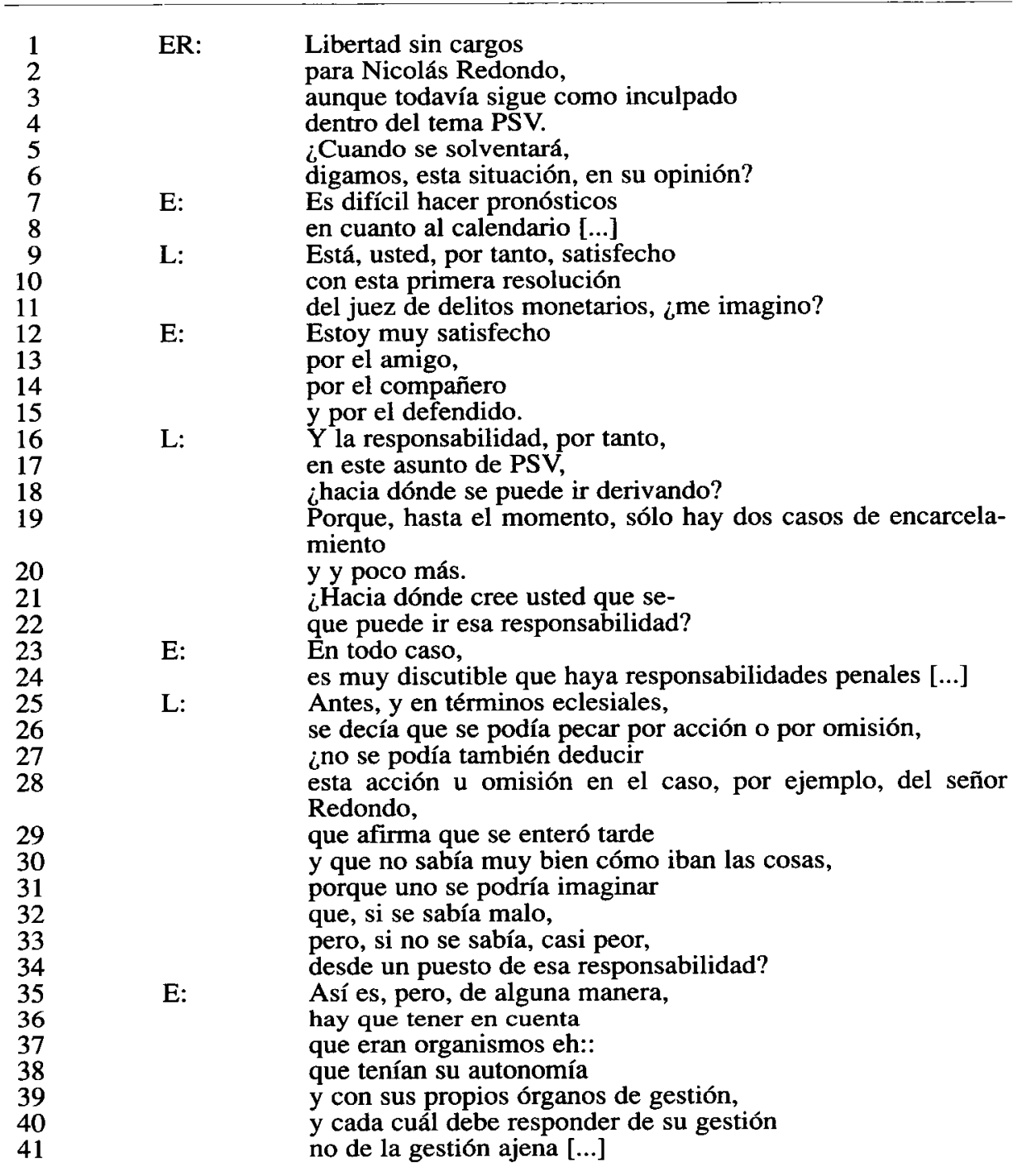

El tema que se trata en la entrevista afecta a uno de los más conocidos líderes sindicales en la época de la transición política española, un personaje además muy respetado dentro de los movimientos de izquierda. El presunto fraude que se menciona es, pues, un asunto enormemente delicado y comprometido para la vida sindical de ese momento. La entrevista se produce a raíz de la noticia que explica cómo Nicolás Redondo, después de prestar declaración ante el Juez por este hecho, ha quedado en libertad sin cargos.

En las primeras preguntas, el ER alude a diferentes aspectos de este caso -líneas 5-6, 9-11, 16-18 y 21-22 - que el EO intenta responder 
(se ha omitido el grueso de estas respuestas porque no resulta relevante para el punto concreto que estamos tratando aquí). A partir de la línea 25, el ER introduce una comparación un tanto inusitada en el contexto en el que surge. El ER se está refiriendo a una persona de la izquierda, así como lo es también el $\mathrm{EO}$, y precisamente el término figurativo o dominio de origen de la comparación que utiliza es uno de los aspectos considerados, desde posiciones de izquierda, menos progresistas de la religión católica, el del pecado.

Este recurso figurativo sirve al ER de prefacio para preparar la alusión al punto más delicado de todo este asunto en relación con Nicolás Redondo: cómo es que este fraude le pasó desapercibido siendo, como era, el Secretario General de ese sindicato en aquel momento. Con la comparación aludida, el ER está reduciendo la fuerza ilocutiva de su pregunta al situar el tema más controvertido dentro de un marco cognitivo con el que el EO no se identifica y, por tanto, puede ser fácilmente refutado. Con ello, consigue reducir también la amenaza que hubiera podido derivarse para su imagen, para la imagen del medio público que representa e incluso también para la integridad de una institución democrática como es un sindicato.

\subsection{Deslegitimación}

El par legitimación/deslegitimación constituye, según Van Dijk (1998: cap. 26), un acto social complejo cuya finalidad es o bien la justificación de las acciones realizadas por determinadas instituciones (legitimación), o bien la depreciación de las acciones del oponente o adversario (deslegitimación). Estas acciones de legitimación y deslegitimación implican principalmente operaciones discursivas encaminadas a reforzar, por un lado, el poder o la autoridad de una institución o grupo, por medio de la referencia a recursos legales, jurídicos o sociales asociados con la autoridad que ostentan, y, por otro lado, a menoscabar el poder o la autoridad que otros grupos oponentes pretenden también consolidar.

En consecuencia, debido a la relación de las operaciones de legitimación/deslegitimación con las instituciones, como hemos indicado, cualquier construcción discursiva orientada a esta finalidad conlleva inevitablemente connotaciones políticas; como señala Van Dijk (1998: 
256), «aquellos que esperamos lleven a cabo actos de legitimación [o deslegitimación] son los que ocupan o han sido designados para un cargo público y ejercen el poder como consecuencia de tal cargo».

En el contexto del medio público que estamos analizando, se destacan especialmente las prácticas discursivas orientadas a la deslegitimación de ciertas actuaciones de otras instituciones o de determinados individuos que han constituido una amenaza para los intereses del partido gobernante. El análisis de esta función va a constituir el segundo bloque de los ejemplos. Veamos, pues, estos ejemplos y las estrategias que se construyen para tal fin.

\subsubsection{Topicalización}

Una de las funciones básicas de las lenguas es la distribución de la información a lo largo de la cadena hablada, según esta información sea más o menos relevante para los interlocutores en la actividad comunicativa. Esta posibilidad de resaltar una determinada información sobre el resto puede tener una finalidad netamente pragmática, pero también puede ser explotada por los interlocutores con fines persuasivos o ideológicos (Van Dijk, 1995: 27-28).

El resultado es una estrategia que presenta una noticia determinada de una manera que discursivamente contrasta con la exposición de otras y que revela una cierta intencionalidad en la forma como se quiere resaltar. Así, en el ejemplo 4, la manera tan explícita como el ER presenta la noticia (la que va a servir de pie a la entrevista) contrasta con otras noticias llevadas a cabo por el ER en otros momentos (véase el ejemplo 1).

\section{EJEMPLO 4}

Entrevista a Pascual Sala, Presidente del Consejo General del Poder Judicial, en la presentación del balance anual sobre la actuación de este organismo. La noticia coincide con un momento en el que los tribunales de justicia han abierto varios sumarios sobre hechos de corrupción en donde se implica a varios personajes públicos ligados al Partido Socialista en el poder (14-9-95).

\begin{tabular}{|c|c|}
\hline ER: & $\begin{array}{l}\text { La verdad que::, } \\
\text { en esta memoria del año, } \\
\text { imagino yo } \\
\text { que también habrá, }\end{array}$ \\
\hline
\end{tabular}




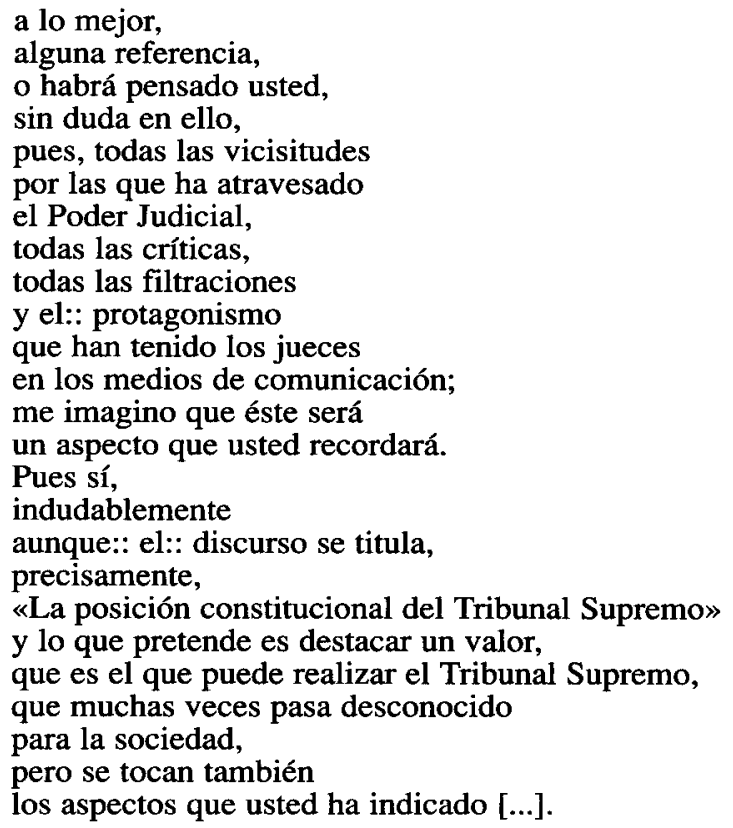

En el primer turno, el ER se refiere a unos hechos sobre el Consejo que el Sr. Sala preside: «todas las vicisitudes por las que ha atravesado el Poder Judicial», en las líneas 9-11. El ER continúa enumerando toda la lista de tales vicisitudes en las líneas 12-16: críticas, filtraciones y protagonismo de los jueces. Esta enumeración puede considerarse una especie de hipotiposis, una figura retórica que proporciona una descripción vívida y pictórica de un hecho real.

Esta descripción queda reforzada lingüísticamente por el uso del artículo definido en todos los elementos enumerados. La utilización del determinante definido contrasta con la del determinante indefinido en la pregunta del ER en el ejemplo 1: «¿Tenía ... algún problema ...?» La repetición anafórica del determinante todas en las unidades informativas 12 y 13 es también un mecanismo formal que aumenta el énfasis de las palabras del ER.

Debido a la posible amenaza que esta explicitud de los hechos puede representar para la imagen del ER, éste reduce la fuerza del acto de habla que ha construido por medio de varios atenuadores: los modificadores la verdad que en la línea 1 y a lo mejor en la línea 5; la expresión imagino yo en la línea 3; y el marcador discursivo pues, línea 9. Con la presentación de esta noticia bajo la apariencia de duda, el ER parece estar protegiendo su imagen ante la respuesta del EO, 
dado que éste representa uno de los máximos órganos del Poder Judicial.

La topicalización que el ER ha construido contrasta también con la respuesta del EO. Este último evita cualquier referencia explícita a los hechos controvertidos que el ER cita. Así, el Juez realiza un cambio de marco o de alianzas (change of footing en el sentido de Goffman, 1981) llevando la discusión hacia términos más generales sobre la función de Tribunal Supremo en un estado democrático - líneas 2127-. Este cambio de marco le permite al EO construir su respuesta sin necesidad de estar constreñido en la dirección de la pregunta del ER. Al final de su intervención, en las líneas 28-29, el Juez confirma brevemente cómo su discurso tratará también sobre los temas que el ER mencionaba en las líneas 9-16; sin embargo, el Juez usa un término más neutro y general para referirse a estos problemas: aspectos, en la línea 29.

El análisis muestra, pues, cómo la intención expresa del ER es resaltar unas acciones ciertamente más negativas que positivas de uno de los órganos más importantes de cualquier régimen democrático, el del Poder Judicial. Sin una explicación adicional que explique el porqué de tales hechos inusitados en una entidad de tal alto rango, el ER ofrece una imagen deslegitimada de tal institución ante la audiencia común. Esta función queda corroborada por la cautela, discursivamente hablando, con la que trata a otras instituciones implicadas también en actividades cuanto menos controvertidas (tal como hemos indicado en los ejemplos 1 y 3 ).

Esta actitud del ER frente a las actividades del Poder Judicial coincide con una serie de críticas que se estaban produciendo en aquel momento en la arena política española, por parte del Gobierno y determinados miembros del partido del Gobierno (el Partido Socialista), consecuencia de la actuación de determinados jueces que investigaban presuntos delitos de corrupción en los que se implicaba a diversos altos cargos socialistas.

\subsubsection{Narrativización de los hechos}

En la presentación discursiva de ciertos hechos, el hablante puede elegir diversos estilos retóricos para referirse al estado concreto de 
cosas que quiere aludir. Entre estos estilos, uno de los más utilizados en el discurso público, como demuestra la tradición retórica, es la técnica narrativa. La función de la narratio consiste en «la exposición o bien de una cosa sucedida o bien tenida por tal, útil para persuadir» (Quintiliano, Institutio oratoria, IV/2/31; véase referencia en Pujante, 1996: 70). En este sentido, el orador no presenta meramente una simple reproducción de los hechos, sino una construcción significativa de los mismos.

En los datos analizados, esta técnica aparece varias veces ligada a un único tema: la referencia a una serie de figuras públicas que en ese momento estaban siendo investigadas por los jueces por su relación con delitos de corrupción. De tales ejemplos, hemos seleccionado el siguiente fragmento:

\section{EJEMPLO 5}

Entrevista a Álvaro Cuesta, representante del Partido Socialista en la «Comisión Luis Roldán», en el Parlamento. Esta comisión investigaba el enriquecimiento injustificado de un ex-alto cargo policial, el Sr. Luis Roldán (1-6-1994).

ER:

[...] Sí, y entonces los puntos de acuerdo están justamente en conocer el que ya se sabe:: cuáles fuecuál fue el sistema de enriquecimiento del señor Roldán.

IE: Eh:: sí, vamos, la relación de hechos eh:: pues apunta a una serie de:: eh::

irregularidades en la gestión, [...]

IR: Señor Cuesta, parece que Lerma, el Presidente de la Comisión Josep López de Lerma decía que podría haber incluso más, responsabilidades penales;

¿hacia dónde se podían extender esas responsabilidades?

Señor Cuesta, eh::

hay una información que aparece hoy:: en-

a través de una emisora de radio y a través también de una agencia de prensa; se diría o se dice que Luis Roldán ha escrito mediante otra persona y:: dice que no se va a presentar nunca, que no va a volver, que se va a cambiar incluso:: de cara, va a cambiar la fisonomía para empezar desde cero una nueva vida. Pero, eso sí, dice que no va a enviaro que no va a destapar más cosas. ¿Cree usted que que tiene fiabilidad esta información y que el Señor Roldán 


$\begin{array}{lll}32 & & \text { definitivamente va a iniciar ese nuevo camino? } \\ 33 & \text { IE: } & \text { Cualquier día cambia de sexo, } \\ 34 & & \text { si seguimos con la } \\ 35 & & \text { de la rumorología ¿no? } \\ 36 & & \text { La verdad es que- } \\ 37 & & \text { yo creo que son especulaciones eh } \\ 38 & & \text { tan gratuitas como:: la de suponer si:: } \\ 39 & & \text { al final va a ser un travestido también, ¿no? }\end{array}$

Desde el comienzo de la entrevista hasta la línea 15, tanto en las preguntas del ER como en las respuestas del EO, la realización discursiva del tema que ambos nos ofrecen es la de una mera alusión denotativa a unos hechos presuntamente ocurridos, que están siendo investigados por una comisión en el Parlamento. Sin embargo, la última pregunta del ER supone un cambio en la exposición de tales hechos, por medio de una presentación de lo sucedido a modo de narración. Así el ER se refiere a esta persona, huida de la justicia, que ha enviado un comunicado por medio de otra persona en cuyo texto explica sus deseos de comenzar una nueva vida en un lugar desconocido del extranjero, a condición de no revelar la información comprometida que al parecer conoce; es decir, un personaje que, a título individual, intenta negociar sus cuentas con la justicia a cambio de su silencio. En la narración de estos hechos, el ER utiliza una estructura formal trimembre —en las líneas 22-26-, fórmula común en casi todas las historias narrativas populares así como en los discursos construidos siguiendo los cánones de la retórica clásica (Atkinson, 1984: 57ss.; Pujante y Morales López, 1997: 53-54).

Lo que esta narración realiza es una reconstrucción determinada de unos hechos que nos presenta a este personaje público desde una perspectiva determinada. En este caso, la narración parece activar el marco cognitivo de un chantaje de este personaje con esos poderes públicos. Sin embargo, el ER explícitamente se desliga de la autoría de esta narración al resaltar que proviene de otro medio público. La respuesta del EO supone una alianza con este marco de interpretación, aunque avanzando un poco más en la construcción retórica: el Sr. Cuesta construye una ironía, derivando el tema iniciado hacia el mundo del travestismo. Así pues, la deslegitimación se completa con la alianza entre los dos interlocutores.

Sin embargo, en aquel momento era bien conocido por la opinión pública todo lo relativo a este asunto: que Luis Roldán se había fugado al saberse buscado por la policía y se había refugiado en un lugar del extranjero. Ciertos rumores indicaban también que se habían producido 
ciertos contactos entre el Gobierno y Luis Roldán con el fin de negociar las condiciones de su entrega. Además, se iba revelando poco a poco que el asunto judicial que concernía a este personaje no era un negocio individual suyo, sino una trama de corrupción en el que estaban implicadas otras personas públicas.

Por tanto, lo que el ER consigue con la utilización de la técnica narrativa es un constructo simplificado y sesgado de unos hechos delictivos, con el que se atribuyen responsabilidades a una persona concreta, obviando la participación y colaboración de otros personajes en tales hechos. De esta forma, se consigue velar discursivamente todo el entramado que ha generado tales hechos, deslegitimando ante la audiencia a un solo personaje.

\subsubsection{Figuras retóricas}

Como ya hemos indicado anteriormente, una gran parte de la atención de los estudios semántico-pragmáticos se ha centrado, en los últimos años, en la investigación de las bases cognitivas de la metáfora, intentando explicar la gran utilización de este recurso no solamente en el discurso literario, sino en la interacción cotidiana y en el discurso de tipo persuasivo. A continuación, mostramos la utilización por parte del ER de una serie de metáforas lexicalizadas con una intención ideológica obvia.

\section{EJEMPLO 6}

Entrevista a Pedro Solbes, Ministro de Economía y Hacienda. El tema se refiere al informe sobre la economía española elaborado por el Fondo Monetario Internacional, en su última reunión en Madrid (4-10-94).

ER: $\quad[\ldots]$ Una asamblea que va a poner punto final a toda una semana de contactos, de reuniones y también eh:: de recetas, como las que se han rechazado para España; pero ¿qué otra cosa ha podido aportar

este encuentro financiero de Madrid, señor Ministro?

EO: $\quad$ Yo creo que hay bastantes elementos de interés

en la discusión de la semana [...] Las críticas que han lanzado enreferidas a nuestro país, a nuestro sistema de pensiones, 
también esas recetas

para crear empleo;

en su opinión, bueno, ¿por qué cree usted que se han lanzado por este camino?

¿es que no hay otra alternativa para estas instituciones,

EO: $\quad$ Hay una vieja polémica

sobre cómo debe tratarse el problema del empleo,

ER: $\quad$ Señor Ministro,

también quisiera preguntarle por la última receta del Banco Mundial,

que en algún momento da la sensación de que es algo que ya hemos oído también aquí en España;

en el momento en que se crea una cierta confusión sobre el futuro de las pensiones, se dice

que podría ser un sistema mixto,

un sistema que el Estado garantice las pensiones a los más pobres;

pero que después habría que completar el resto de pensiones con el- digamos, con capital privado.

Este segundo balance

o esta recomendación del Banco Mundial

EO: $\quad$ ¿le suena a usted mejor, señor Solbes? sistema público y sistema eh:: privado;

es decir, están haciendo una receta

en lo que ellos llaman los tres pilares [...]

ER: $\quad$ Señor Solbes, parece que las recetas que suelen dar o que estaban dando en estos- en estas oportunidades

el Fondo Monetario y el Banco Mundial son recetas para ricos,

que evidentemente serían los que no necesitasen

EO: $\quad$ Es- Ese es el elemento ¿no?

es decir, es verdad que nosotros pensamos

que un esquema en el que eh

la aportación se realice al fondo único,

propiedad, o mejor dicho, gestionado por el sector público, la garantía de solidaridad es mayor [...]

El ER realiza una serie de preguntas al ministro sobre las conclusiones que el Fondo Monetario Internacional y el Banco Mundial han elaborado sobre la economía española; sin embargo, al mismo tiempo el ER nos ofrece su propia valoración -o la valoración del «autor» de este medio público, en el sentido de Goffman (1981: 144-145)—, sobre el hecho en cuestión. Esta opinión propia no se produce de manera directa, sino a través de la utilización de varias metáforas lexicalizadas.

En primer lugar, se usa el término «recetas»-que se repite varias veces (líneas 3, 13, 22 y 36)—, para referirse a las propuestas que el Fondo propone para mejorar nuestra economía; asimismo, el predicado «lanzar», en la línea 15, para indicar la orientación económica 
que ofrecen para España. En uno de los casos (línea 31), en el que usa términos más denotativos («balance» y «recomendación»), el ER traslada la metáfora lexicalizada al predicado (en este caso, «sonar»): «Este segundo balance o esta recomendación del Banco Mundial ile suena a usted mejor, señor Solbes?».

En contraste con el ER, las diferentes respuestas del Ministro se caracterizan por la utilización de términos denotativos, de los cuales no parece interpretarse significado inferido (solamente hay una excepción, la de la línea 34, en donde Solbes repite el término «receta» de la pregunta). En general, pues, en las respuestas del Ministro, se destaca la prudencia de sus valoraciones sobre el Fondo Monetario y el Banco Mundial (a pesar de que, como socialista, podría esperarse una cierta oposición a estos organismos), que contrasta con las prácticas discursivas del ER.

Éste, a través de la utilización de las metáforas señaladas, construye un marco interpretativo cuya función es la de deslegitimar la actuación del Fondo Monetario y sus propuestas económicas para nuestro país. Estas metáforas lexicalizadas le permiten construir un marco de conocimiento específico a partir de la atribución de determinados rasgos del término figurado o «dominio de origen» (source domain, si utilizamos la terminología de Lakoff y Johnson 1980; Lakoff, 1987: cap. 17) al término real o «dominio meta» (target domain). Las palabras «recetas» y «lanzarse» activan marcos cognitivos con rasgos tales como los de «simplificación», «improvisación» o «falta de profundidad en sus propuestas», para calificar las recomendaciones económicas de estos organismos. La activación de estas características es lo que, en esta entrevista, contribuye a la deslegitimación de estos dos organismos internacionales; con ello se consigue también legitimar, de paso, la actitud de las autoridades españolas que han criticado la actuación de tales organismos internacionales.

\section{CONSIDERACIONES FINALES}

El análisis presentado en el apartado anterior ha mostrado diferentes prácticas simbólicas a través de las cuales el periodista, a lo largo del desarrollo de las diferentes entrevistas, construye su rol social de entrevistador de un medio de comunicación de titularidad pública. En consecuencia, desde el momento que resaltamos que este hablante representa un rol social determinado, sus palabras necesitan ser anali- 
zadas desde un trasfondo sociocultural concreto (Bakhtin, 1981: 110; Verschueren, 1995: 132). Cuando, además, nos encontramos con que este sujeto social habla en nombre de una institución que representa una estructura de poder determinada, el análisis necesita desvelar también el posible contenido ideológico implícito. Con todo, como señala Thompson (1990: 56), estos mecanismos lingüístico-discursivos no son portadores por sí mismos de ideología, sino que son solamente portadores de contenidos ideológicos en la medida en que, en circunstancias concretas, sirven a sus creadores para conseguir fines relacionados con determinadas situaciones de poder.

En los ejemplos mostrados, hemos podido constatar cómo la construcción de una serie de prácticas simbólicas permiten al entrevistador transmitir, vía inferencias, la intencionalidad comunicativo-social de él mismo o del autor del medio público que representa. Las razones por las cuales el entrevistador se ve impelido al uso de tales técnicas indirectas se pueden resumir en las siguientes: la dificultad de aludir a algunos de los temas, como consecuencia de su relevancia política o social; el deseo de transmitir una determinada posición ante los hechos, aunque bajo la apariencia de neutralidad; y la crítica a actuaciones de otros cargos públicos o instituciones con los cuales no se está de acuerdo.

A lo largo del apartado de análisis hemos intentado demostrar cómo estas intenciones inmediatas que el entrevistador consigue a través de las diferentes estrategias discursivas que ha construido cumplen dos funciones sociales más amplias; por un lado, a la defensa de la imagen del entrevistador, en tanto portavoz de una institución de carácter público, que quiere realizar el difícil juego de equilibrio entre libertad de expresión y defensa de los intereses del Gobierno y de ciertas instituciones democráticas. Por otro lado, se realiza una labor de deslegitimación de otras instituciones con cuyos intereses no se coincide y de determinados personajes públicos envueltos en procesos judiciales a quienes se les parece atribuir el peso de una responsabilidad que en realidad tienen que ser compartida también por otros.

\section{Referencias bibliográficas}

AgAR, M. (1985). «Institutional discourse». Text 5/3, 147-168.

ATKINSON, M. (1984). Our masters' voices: The language and body language of politics. London: Methuen. 
BAKHTIN, M. (1981). Teoría y estética de la novela. Madrid: Taurus, 1989.

BAlsebre, A.; MATEU, M., y VIDAL, D. (1998). La entrevista en radio, televisión y prensa. Madrid: Cátedra.

BuSTAMANTE, E. (1989). «TV and public service in Spain: A difficult encounter» Media, Culture and Society 11, 67-87.

CantaVella, J. (1996). Manual de la entrevista periodística. Barcelona: Ariel.

Clayman, St. E. (1992). «Footing in the achievement of neutrality: The case of news-interview discourse». En Drew, P. y Heritage J. (eds.) (1992), 163-198.

ChILTON, P. e IlyIN, M. (1993). «Metaphor in political discourse: The case of the "common European house"». Discourse and Society 4/1, 7-31.

DREw, P. y HeRITAGE, J. (1992). Talk at work. Cambridge University Cambridge: Press.

DURANTI, A. (1994). From grammar to politics. Linguistic anthropology in a Western Samoan village. Los Ángeles: University of California Press.

FAIRCLOUGH, N. (1989). Language and power. London: Longman.

FouCAult, M. (1970). El orden del discurso. Barcelona: Tusquets, 1973.

GoFfMAN, E. (1971). Relaciones en público. Microestudios de orden público. Madrid: Alianza Editorial, 1979.

- (1974). Frame analysis. New York: Harper \& Row.

- (1981). Forms of talk. Philadelphia: University of Pennsylvania Press.

GUMPERZ, J. J. (1982). Discourse strategies. Cambridge: Cambridge University Press.

HALL, S. (1973). "A world at one with itself». En The manufacture of news. Social problems, deviance and mass media, S. COHEN y J. YounG (eds.), 8594. London: Constable.

HeRITAGE, J. C., y GreatBATCH, D. (1991). «On the institutional character of institutional talk: The case of news interviews». En Talk and social structure, D. BODEN y D. H. ZIMMERMAN (eds.), 93-137. Cambridge: Polity Press.

HeRITAGE, J. C., y Roth, A. L. (1995). «Grammar and institution: Questions and questioning in the broadcast of news interview». Research on Language and Social Interaction 28/1, 1-60.

KUMAR, K. (1975). «Holding the middle ground: The BBC, the public and the professional broadcaster». Sociology 9, 67-88.

LAKOFF, G. (1987). Women, fire and dangerous things. What categories reveal about the mind. Chicago: The University of Chicago Press.

LAKOFF, G., y JoHnson, M. (1980). Metáforas de la vida cotidiana. Madrid: Cátedra, 1986.

Levinson, St. C. (1983). Pragmática. Barcelona: Teide, 1989. - (1992). «Activity types and language» En DREw, P. y J. Heritage (eds). (1992), 66-100.

MORALES LÓPEZ, E. (1995). «Buscando la neutralidad: Un análisis contrastivo de dos entrevistas radiofónicas» Lingüística Española Actual XVII/1, 107-125.

- (1998). «Spanish public radio: Fictionalization of events in the presentation of conflicting news». [Trabajo presentado en el 6th International Pragmatic Conference, Reims (Francia)]. 
PRESENTACIÓN DE LA INFORMACIÓN EN ENTREVISTAS DE LA RADIO PÚBLICA...

PUjANTE, D., (1996). El hijo de la persuasión. Quintiliano y el estatuto retórico. Logroño: Instituto de Estudios Riojanos.

Pujante, D., y Morales LóPez, E. (1997). «Discurso político en la actual democracia española». Discurso 21/22, 39-75.

SCHANK, R. C., y ABELSON, R. P., (1977). Guiones, planes, metas y entendimiento. Barcelona: Paidós, 1987.

ThOMPSON, J. B. (1990). Ideology and modern culture. Cambridge: Polity Press.

VAN DiJK, T. A. (1995). «Discourse analysis as ideology analysis». En Language and peace, C. SCHÄFFNER y A. L. WENDEN (eds.), 17-33. Aldershot: Dartmouth.

- (1998). Ideology. A multidisciplinary approach. London: Sage.

VERSCHUEREN, J., (1995). «The pragmatics return to meaning: Notes on the dynamics of communication, degrees of salience, and communicative transparency». Journal of Linguistic Anthropology 5/2, 127-156. 